\section{Targeting CDK in triple-negative breast cancer}

\author{
By Kai-Jye Lou, Staff Writer
}

A team at the University of California, San Francisco has found a dysregulated MYC pathway that underlies the majority of triple-negative breast cancers-a form of the disease with limited treatment options. ${ }^{1}$ The researchers also showed that inhibitors of cyclin dependent kinase, a target upon which MYC-upregulated cancers are dependent, could selectively kill the triple-negative cancer cells.

The group is planning a clinical trial of an undisclosed cyclin dependent kinase (CDK) inhibitor.

Triple-negative breast cancers do not express estrogen receptor, progesterone receptor and HER2 (EGFR2; ERBB2; neu). These tumors are thus insensitive to many of the treatments for other breast cancer subtypes, such as hormone ablation and Herceptin trastuzumab, a humanized antiHER2 $\mathrm{mAb}$ marketed by Roche and its Genentech Inc. unit.

Due to the lack of well-defined targets, developing targeted therapies against triple-negative disease has been challenging. There are no approved targeted therapies for triple-negative breast cancer, and standard care remains chemotherapy.

Andrei Goga, an associate professor of medicine at UCSF, has been tackling the indication by looking for targets in oncogenic pathways outside of the hormone receptors and HER2.

In 2007, Goga and colleagues reported that several other types of cancer with high expression of MYC (c-Myc) become dependent on survivin (BIRC5), an inhibitor of apoptosis that is rapidly degraded in the absence of CDK1 (CDC2) activity. ${ }^{2}$ The team also showed that small molecule CDK1 inhibitors could exploit this vulnerability to selectively kill survivin-dependent cancer cell lines. ${ }^{3}$

The following year, a different group reported that about $70 \%$ of triple-negative breast tumors are classified as basal-like, which often has an aggressive phenotype. ${ }^{4}$

The final puzzle piece came from multiple other groups that showed MYC plays an important role in aggressive breast cancers, although those groups did not look specifically at triple-negative breast cancers. ${ }^{5}$

Thus, Goga's group sought to determine whether the MYC pathway was at work in triple-negative breast cancer, and if so whether such tumors would be sensitive to CDK inhibitors.

In primary breast tumor samples, triple-negative samples showed significantly higher expression of MYC mRNA and protein than receptor-positive samples $(p<0.0001$ and $p<0.04)$. In a panel of
50 human breast cancer cell lines, MYC mRNA expression in the 25 triple-negative lines was greater than that in the non-triple-negative lines $(p<0.01)$.

Moreover, patients who had more MYC activity had significantly lower disease-free survival and had early disease recurrence ( $p=0.005$ and $p=0.001$ ).

With the link established between triple-negative breast cancer, increased MYC activity and poor disease prognosis, the researchers next sought to determine whether their earlier findings with CDK inhibitors in other MYC-upregulated cancers applied to this disease subgroup.

In five triple-negative human breast cancer cell lines, the small molecule CDK inhibitors purvalanol A and dinaciclib decreased cell growth compared with vehicle. In mouse xenograft models of triplenegative human breast cancer, twice-weekly dinaciclib for two weeks resulted in tumor regression.

The effect of $\mathrm{CDK}$ inhibition was dependent on the upregulation of BCL2-like 11 apoptosis facilitator (BCL2L11; BIM), which is known to promote apoptosis. ${ }^{6}$

Purvalanol A is a research reagent. Dinaciclib from Merck \& Co. Inc. and Ligand Pharmaceuticals Inc. is in Phase II testing to treat multiple hematological cancers and non-small cell lung cancer (NSCLC). The compound is not being tested in breast cancer.

Results were published in The Journal of Experimental Medicine. Merck declined to comment, and Ligand did not respond to requests for an interview.

"The publication adds to previous data highlighting a possible synthetic lethal relationship between the MYC oncoprotein and cyclin dependent kinase enzymes in a variety of tumor types," said Sheelagh Frame, seliciclib program manager at Cyclacel Pharmaceuticals Inc. "However, their analysis and interpretation is somewhat limited to the basal subtype of triple-negative breast cancer, where it has previously been reported that the
"The publication adds to previous data highlighting a possible synthetic lethal relationship between the MYC oncoprotein and cyclin dependent kinase enzymes in a variety of tumor types."

-Sheelagh Frame, Cyclacel Pharmaceuticals Inc.
MYC pathway signature shows a strong correlation with basal-like histology. Future work would need to address whether MYC is disproportionately upregulated in the other subtypes of triple-negative breast cancer."

Cyclacel's first-generation small molecule CDK inhibitor seliciclib is in Phase II testing for nasopharyngeal cancer and NSCLC. The company's second-generation inhibitor, CYC065, is in preclinical development for cancer.

Dinaciclib inhibits CDK1, CDK2, CDK5 and CDK9, whereas seliciclib inhibits CDK2, CDK5, CDK7 and CDK9. Purvalanol A inhibits CDK1 and CDK2.

\section{Quick moves to the clinic}

As multiple companies already are testing CDK inhibitors in the clinic to treat other types of cancer, Goga said the clear next step is to run a clinical 
study in triple-negative breast cancer. His group is planning a trial, but further details were not disclosed.

He did say it will be important to determine whether having a companion molecular diagnostic for assessing MYC abundance would be useful in such a trial.

Goga also noted that CDK inhibitors might have a synergistic effect with inhibitors of poly(ADP-ribose) polymerase (PARP), one of the few targets companies are pursuing for triple-negative breast cancer.

PARP inhibitors could use the boost because despite their initial promise, this class of compounds has encountered a string of setbacks in the clinic that have led some to question the target's relevance in triple-negative tumors.

In 2011, Sanofi's PARP inhibitor iniparib failed in a Phase III trial, and a study later that year from researchers at Abbott Laboratories suggested that iniparib's primary mechanism of action is not PARP inhibition. ${ }^{7}$

In 2010, AstraZeneca plc closed recruitment of patients with triplenegative breast cancer in a Phase I/II trial of its PARP inhibitor olaparib after noting that this particular cohort did not meet continuation criteria.

Although Abbott's PARP inhibitor veliparib still is being tested in patients who have triple-negative breast cancer, data from a Phase II trial in 2009 suggested the compound's effect would be limited to the small percentage of patients who also carry a mutation in the breast cancer 1 early onset (BRCA1) or BRCA2 gene. ${ }^{8,9}$

Goga noted that CDK inhibitors have the potential to expand the utility of PARP inhibitors beyond patients with mutated $B R C A$, as a group at Dana-Farber Cancer Institute reported last year that Cdk1 inhibition sensitized mice with wild-type Brca tumors to PARP inhibitors. ${ }^{10}$

If the Dana-Farber findings translate to the triple-negative breast cancer setting, the results would suggest that CDK inhibitors could expand the use of PARP inhibitors to the greater population of triplenegative patients who also express wild-type $B R C A$.

Cyclacel's Frame added that the researchers should carry out studies to determine the relationship between different levels of MYC expression and sensitivity to CDK inhibition. Moreover, she also wanted to know whether there is an association between the levels of MYC and cyclin E (CCNE) expression in the primary patient samples.
"Previous reports have indicated that overexpression of cyclin $\mathrm{E}$ is frequently found in triple-negative tumors and may confer hypersensitivity to small molecule inhibitors of CDK2, such as seliciclib," she told SciBX.

On the preclinical side, Goga's group is investigating the effects of CDK inhibitors in primary triple-negative breast cancer xenograft mouse models and trying to elucidate the mechanism behind BIM upregulation in response to CDK inhibition.

The work described in JEM is unpatented.

Lou, K.-J. SciBX 5(15); doi:10.1038/scibx.2012.379

Published online April 12, 2012

\section{REFERENCES}

1. Horiuchi, D. et al. J. Exp. Med.; published online March 19, 2012; doi:10.1084/jem.20111512

Contact: Andrei Goga, University of California, San Francisco,

Calif.

e-mail: andrei.goga@ucsf.edu

2. O'Connor, D.S. et al. Proc. Natl. Acad. Sci. USA 97, 13103-13107 (2000)

3. Goga, A. et al. Nat. Med. 13, 820-827 (2007)

4. Bertucci, F. et al. Int. J. Cancer 123, 236-240 (2008)

5. Chen, Y. \& Olopade, O.I. Expert Rev. Anticancer Ther. 8, 1689-1698 (2008)

6. O'Connor, L. et al. EMBO J. 17, 384-395 (1998)

7. Liu, X. et al. Clin. Cancer Res. 18, 510-523 (2012)

8. Isakoff, S.J. et al. J. Clin. Oncol. (Meeting Abstracts) 28, 1019 (2010)

9. Hiller, D.J. \& Chu, Q.D. Int. J. Breast Cancer 2012, 829315; published online Oct. 25, 2011; doi:10.1155/2012/829315

10. Johnson, N. et al. Nat. Med. 17, 875-882 (2011)

\section{COMPANIES AND INSTITUTIONS MENTIONED}

Abbott Laboratories (NYSE:ABT), Abbott Park, III. AstraZeneca plc (LSE:AZN; NYSE:AZN), London, U.K. Cyclacel Pharmaceuticals Inc. (NASDAQ:CYCC), Berkeley Heights, N.J.

Dana-Farber Cancer Institute, Boston, Mass.

Genentech Inc., South San Francisco, Calif.

Ligand Pharmaceuticals Inc. (NASDAQ:LGND), La Jolla, Calif.

Merck \& Co. Inc. (NYSE:MRK), Whitehouse Station, N.J.

Roche (SIX:ROG; OTCQX:RHHBY), Basel, Switzerland

Sanofi (Euronext:SAN; NYSE:SNY), Paris, France

University of California, San Francisco, Calif. 